\title{
The effects of hematocrit and age on transcranial Doppler measurements in patients with recent ischemic stroke
}

\author{
Canan Togay Isikay, Nevzat Uzuner*, Demet Gücüyener*, Gazi Özdemir* \\ Department of Neurology, Ankara University School of Medicine, *Osmangazi University School of Medicine, Turkey
}

\begin{abstract}
Background: The effects of age and hematocrit on transcranial Doppler (TCD) velocities have not been evaluated in a large patient group with recent ischemic stroke. Aim: This study assessed the effects of age and hematocrit on TCD measurements in patients with recent ischemic stroke compared to patients with non-vascular diseases. Settings and Design: University Hospital, retrospective study. Materials and Methods: TCD records and data files of 862 consecutive patients (mean age, $57 \pm 16$ years) with various neurological diagnoses were reviewed retrospectively. The peak systolic, end diastolic and mean flow velocities (FV), systolic/diastolic ratios and pulsatility indices $(\mathrm{PI})$ in the middle cerebral arteries were averaged and the effect of age and hematocrit values on these TCD values was studied. Independent samples $t$ test, Pearson's coefficients of correlation, and linear regression test were used for statistical analysis. Results: Among 862 patients, 413 were women (mean age, $53 \pm 17$ years) and 449 were men (mean age, 60 \pm 13 years). Peak systolic and mean FV were higher and hematocrit concentration was lower in women compared to men $(P<0.001)$. The relation of TCD velocities with age and hematocrit was more remarkable in the group of patients with non-vascular neurological disorders. PI values demonstrated a significant correlation to age $(r=+0.47)$ $(P<0.001)$, but did not change significantly with hematocrit level. Conclusions: It should be remembered that blood FV measured by TCD may be significantly affected by age and hematocrit level. However, there seems to be no significant association between TCD velocities and hematocrit in patients with a recent ischemic stroke.
\end{abstract}

Key Words: Transcranial Doppler, hematocrit, stroke

\section{Introduction}

Transcranial Doppler (TCD) is a non-invasive, safe and easy method to measure blood flow velocities (BFV) in the basal cerebral arteries. It is widely used for the evaluation of patients with ischemic stroke, subarachnoid hemorrhage and sickle cell anemia. However, TCD measurements can be significantly affected by some individual and physiological factors. The major hemodynamic factors determining BFV in the basal cerebral arteries are the length and cross-sectional area of the vessel, pressure gradient along a vessel, and viscosity of the blood. ${ }^{[1]}$ Other factors associated with cerebral BFV include age and sex of the subject, ${ }^{[2,3]}$ partial pressure of oxygen and carbon dioxide, ${ }^{[4,5]}$ body temperature, ${ }^{[6]}$ and some physiological changes, such as sleeping or awakening and exercise. $^{[7,8]}$

Previous reports demonstrated that age was associated with decreasing flow velocities and increasing pulsatility indices. ${ }^{[2-}$ ${ }^{4,9,10]} \mathrm{An}$ average decline of $0.3-0.5 \%$ per year in the mean flow velocities (MFV) was suggested in the individuals between 20-70 years of age. ${ }^{[11]}$

Hematocrit is the major determinant of blood viscosity, and cerebral blood flow was shown to be inversely correlated with the hematocrit level. ${ }^{[12,13]}$ These studies were followed by others performed with TCD in small patient groups. ${ }^{[1,9,14]}$ The correlation of TCD parameters with hematocrit concentration has not been evaluated previously in a population with recent stroke.

The aim of this study was to assess the influence of hematocrit level and age on TCD measurements in a larger group of patients with ischemic stroke or other non-vascular neurological disorders.

\section{Materials and Methods}

Complete data of the patients who were referred to the Neurovascular Laboratory between January 1996 and September 2001 were reviewed retrospectively (n:1500). Patients with complete TCD examination that was consisted of BFV in all basal brain vessels and performed on the day of performing blood tests were selected. Patients with evidence of cerebrovascular stenosis or intracranial hemorrhage, those with impaired consciousness, and those with known 
hematological disorders, heart failure, chronic obstructive pulmonary disease or other pulmonary diseases by history or physical examination were excluded. Patients with a significant difference $(>50 \%$ ) of flow velocities between two sides were also excluded. There were 862 records remaining for evaluation. A total of 638 patients were excluded due to missing data, incomplete evaluation and fulfillment of exclusion criteria.

General epidemiological features, diagnoses, blood counts, and all imaging studies available in the patient records were reviewed. TCD examinations were performed in the Neurovascular Laboratory by the same author (Uzuner N, MD, Professor of Neurology), using 2$\mathrm{MHz}$ pulsed-wave Doppler transducer and TCD scanner (DWL Multidop X4, TCD8 software). The average values for peak systolic (PSV), end diastolic (EDV) and mean flow velocities (MFV), pulsatility indices (PI) (peak velocity minus diastolic velocity divided by mean velocity), and systolic/diastolic ( $\mathrm{S} / \mathrm{D})$ ratios in the middle cerebral arteries (insonated through the temporal window at a depth of $35-55 \mathrm{~mm}$ ) were recorded.

Patients were divided into two subgroups; Group 1 consisted of patients with recent ischemic stroke and Group 2 comprised patients with epilepsy, syncope, peripheral vertigo, demyelinative disease, and those with miscellaneous neurological symptoms or signs (ocular motor paresis, facial pain, ptosis etc.) but no defined diagnosis.

We used Student's $t$ and Mann-Whitney U tests to compare age, TCD measurements and hematocrit levels between the two groups and sexes. Pearson's and Spearman's coefficients of correlations were used to determine the association between hematocrit, age and TCD parameters. $P<0.05$ was accepted as statistically significant.

\section{Results}

Among 862 patients (mean age, $57 \pm 16$ years; range, 17 to 95 years), 413 were women (mean age, $53 \pm 17$ years) and 449 were men (mean age, $60 \pm 13$ years). Men were significantly older than women $(P<0.001)$. Neurological diagnoses of the patients are shown in Table 1 . Ischemic stroke was the primary indication for neurovascular examination and was documented in 551 patients $(64 \%)$. Hypertension, diabetes mellitus and smoking were present in $40.8 \%, 15.3 \%$ and $14.8 \%$ patients, respectively.

Laboratory data and TCD measurements including the average values of PSV, EDV, MFV, PI levels and S/D ratios in the middle cerebral arteries (MCA) are shown in Table 2. PSV and MFV were highly correlated $(r=0.93, P<0.001)$, and MFV was inversely correlated with PI values $(r=-0.45$, $P<0.001)$.

\begin{tabular}{lc}
\hline \multicolumn{1}{c}{ Table 1: The diagnoses of patients included in the study } \\
\hline Diagnosis & $\mathbf{n ~ ( \% )}$ \\
Ischemic stroke & $551(63.9)$ \\
Demyelinative diseases & $99(11.5)$ \\
Vertigo* & $90(10.4)$ \\
Syncope & $34(4.0)$ \\
Epilepsy & $27(3.1)$ \\
Miscellaneous & $61(7.1)$ \\
Total & 862 \\
\hline
\end{tabular}

*Patients with vertigo due to ischemic stroke involving posterior circulation were not included in this group.
PSV and MFV in the MCA were higher in women $(P<0.001)$, while hematocrit concentrations were significantly lower in women compared to men $(P<0.001)$ (Table 2$)$. There was no significant association between age and hematocrit. The patients aged over 60 years had significantly lower flow velocities and higher PI values in the MCA compared to those who were younger in age $(P<0.001)$ (Table 2$)$.

Age, TCD measurements, and hematocrit concentrations in the two groups are shown in Table 2. The hematocrit concentration was not significantly different between the two groups. PI values were significantly higher in Group 1, while MFV were lower in Group 1 than in Group $2(P<0.001)$.

Statistical analyses revealed that MFV in the MCA has a weak inverse relation with hematocrit level (rho $=-0.18)$, and a modest inverse relation with age $($ rho $=-0.43)$ (both $P<0.001)$. Age demonstrated a positive correlation with PI level in the MCA $(\mathrm{rho}=+0.55)(P<0.001)$. The inverse correlation between $\mathrm{MFV}$ and age was more notable in Group 2 (rho $=-0.59)$ compared to Group 1 (rho=-0.33). MFV were also more influenced by hematocrit concentration in Group 2 $($ rho $=-0.30)$ than in Group 1 (rho=-0.12). Pulsatility index and S/D were not significantly influenced by hematocrit level $(P=0.09)$. By linear regression analysis, MFV in the MCA was $102.12+(-1.06 x H c t)$ and $88.22+(-0.54 x A g e)$ for patients in Group $2(P<0.001)$ (Figures 1, 2).

\section{Discussion}

The women in our study population had significantly lower hematocrit levels, which was the probable cause of increased flow velocities compared with men. It is important to note that there was no significant association between age and hematocrit.

In this study, BFV were inversely related with age, and distal resistance was higher in the older individuals $(>60$ years of age) than those aged $<60$ years. Age was the only variable that demonstrated a significant correlation to PI, and the strongest inter-individual velocity predictor that was independent of hematocrit concentration. These results are in concordance with the study of Macko et al showing an inverse relation between age and MCA velocities, and a positive correlation of age to PI. ${ }^{[4]}$ Lower MFV and higher PI level in Group 1 can also be explained with the higher mean age of the patients in this group.

A relation between blood viscosity and TCD velocities in the MCA was also reported in previous studies but in smaller patient groups. ${ }^{[1,9,14-17]}$ Brass et al evaluated the effects of hematocrit on TCD-measured BFV in the MCA of 45 patients and showed higher flow velocities in patients with lower hematocrit levels $(r=-0.71){ }^{[1]}$ Ameriso et al evaluated 42 healthy volunteers and reported a modest inverse relationship between hematocrit level and TCD measured-MFV in the MCA $(r=-0.42) .{ }^{[9]}$ Fiermonte et al showed that TCD ve- 


\begin{tabular}{|c|c|c|c|c|c|c|c|c|}
\hline & $n$ & Aqe & PSV $(\mathrm{cm} / \mathrm{s})$ & $\operatorname{EDV}(\mathrm{cm} / \mathrm{s})$ & MFV $(\mathrm{cm} / \mathrm{s})$ & $\mathrm{PI}(\mathrm{cm} / \mathrm{s})$ & $S / D$ & Het (\%) \\
\hline Women & 413 & $53 \pm 17$ & $92 \pm 24$ & $38 \pm 13$ & $60 \pm 18$ & $0.9 \pm 0.2$ & $2.5 \pm 0.6$ & $39 \pm 4$ \\
\hline Men & 449 & $\begin{array}{l}60 \pm 13 \\
P<0.001 \\
u: 53521\end{array}$ & $\begin{array}{l}85 \pm 23 \\
P<0.001 \\
u: 37706\end{array}$ & $\begin{array}{l}32 \pm 11 \\
P<0.001 \\
u: 33935\end{array}$ & $\begin{array}{l}52 \pm 15 \\
P<0.001 \\
u: 33865\end{array}$ & $\begin{array}{l}1.0 \pm 0.2 \\
P<0.001 \\
u: 35842\end{array}$ & $\begin{array}{l}1.0 \pm 0.2 \\
P<0.001 \\
u: 36905\end{array}$ & $\begin{array}{l}42 \pm 4 \\
P<0.001 \\
u: 55323\end{array}$ \\
\hline Age $\leq 60$ & 448 & $45 \pm 11$ & $92 \pm 20$ & $40 \pm 11$ & $60 \pm 15$ & $0.8 \pm 0.1$ & $2.3 \pm 0.4$ & $40 \pm 4$ \\
\hline Age $>60$ & 414 & $70 \pm 7$ & $\begin{array}{l}84 \pm 25 \\
P<0.001 \\
u: 26846\end{array}$ & $\begin{array}{l}29 \pm 10 \\
P<0.001 \\
u: 16580\end{array}$ & $\begin{array}{l}49 \pm 15 \\
P<0.001 \\
t: 8.505 \\
95 \% \quad C l: 8.59-13.75\end{array}$ & $\begin{array}{l}1.1 \pm 0.2 \\
P<0.001 \\
u: 16117\end{array}$ & $\begin{array}{l}1.1 \pm 0.2 \\
P<0.001 \\
u: 15462\end{array}$ & $\begin{array}{l}40 \pm 4 \\
P=0.54 \\
u: 65256\end{array}$ \\
\hline Group 1* & 551 & $61 \pm 13$ & $87 \pm 26$ & $33 \pm 12$ & $53 \pm 17$ & $1.0 \pm 0.3$ & $2.7 \pm 0.8$ & $40 \pm 5$ \\
\hline Group $2 \S$ & 311 & $\begin{array}{l}49 \pm 16 \\
P<0.001 \\
u: 35761\end{array}$ & $\begin{array}{l}91 \pm 21 \\
P=0.12 \\
u: 34127\end{array}$ & $\begin{array}{l}39 \pm 12 \\
P<0.001 \\
u: 27887\end{array}$ & $\begin{array}{l}60 \pm 16 \\
P<0.001 \\
t: 4.377 \\
95 \% \mathrm{Cl}: 3.60-9.46\end{array}$ & $\begin{array}{l}0.9 \pm 0.2 \\
P<0.001 \\
u: 25989\end{array}$ & $\begin{array}{l}2.4 \pm 0.6 \\
P<0.001 \\
u: 26741\end{array}$ & $\begin{array}{l}40 \pm 4 \\
P=0.22 \\
u: 79923\end{array}$ \\
\hline Total & 862 & $57 \pm 16$ & $88 \pm 24$ & $35 \pm 13$ & $55 \pm 17$ & $1.0 \pm 0.3$ & $2.6 \pm 0.8$ & $40 \pm 5$ \\
\hline
\end{tabular}

TCD: Transcranial Doppler, PSV: Peak systolic velocity, EDV: End diastolic velocity, MFV: Mean flow velocity, PI: Pulsatility index, S/D: Systolic/diastolic ratio, Hct: Hematocrit concentration, *Patients with a recent ischemic stroke, § Patients with a non-vascular neurological disorder.

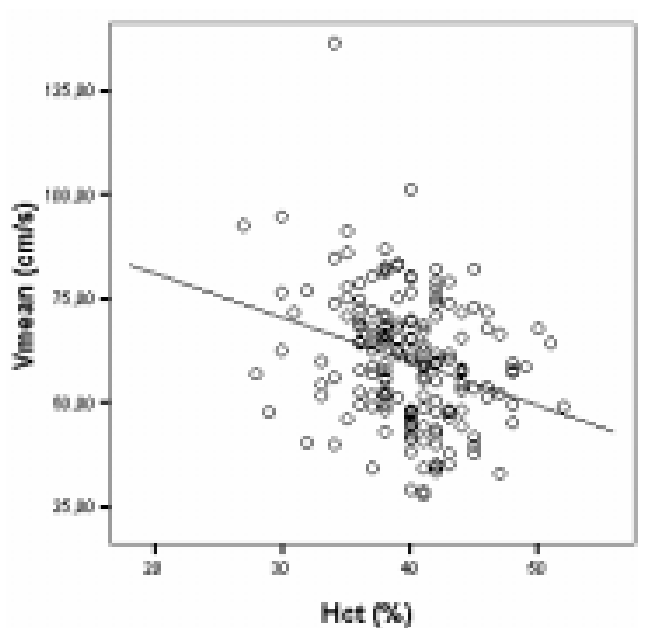

Figure 1: Scatterplot of relation between hematocrit and mean flow velocities in the middle cerebral arteries of the patients in Group 2

locities in patients with polycythemia vera increased significantly after pharmacological and/or phlebotomic treatment. ${ }^{[14]}$ The inverse relation of $\mathrm{BFV}$ with hematocrit can be best explained with the relation of hematocrit to blood viscosity and arterial oxygen content. ${ }^{[9]}$ In this study, a modest inverse correlation was found between hematocrit level and TCD velocities in patients with non-vascular neurological disorders. However, TCD velocities in patients with a recent ischemic stroke were not affected by hematocrit significantly. The relation of TCD velocities with age was also more notable in patients with non-vascular neurological disorders than the others. We think that the lesser impact of hematocrit level and age on TCD velocities in patients with a recent ischemic stroke may be due to impaired autoregulation of the cerebral vessels.

Transcranial Doppler of the basal cerebral arteries is widely used to evaluate the patients with subarachnoid hemorrhage, sickle cell anemia, and intracranial hypertension. TCD analysis in these patients may become challenging if they have significant anemia or polycythemia. Increased TCD velocities compatible with moderate to severe vasospasm might be mim-

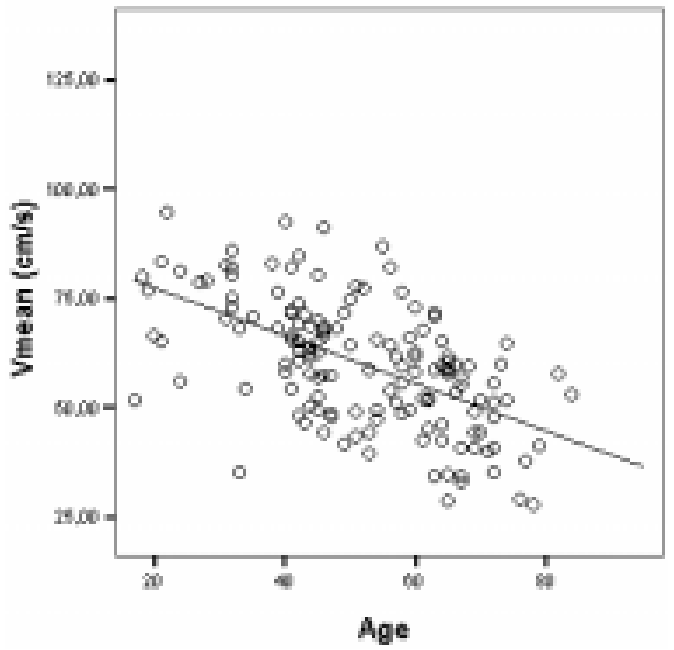

Figure 2: Scatterplot of relation between age and mean flow velocities in the middle cerebral arteries of the patients in Group 2

icked by significant anemia. On the other hand, normal flow velocities in patients with polycythemia can be interpreted falsely negative. Our observations indicate that corrections of TCD velocities may be required for elderly patients and for patients with significant anemia or polycythemia. However, a similar correction does not seem to be necessary in patients with ischemic stroke.

\section{References}

1. Brass LM, Pavlakis SG, DeVivo D, Piomelli S, Mohr JP. Transcranial Doppler measurements of the middle cerebral artery. Effect of Hematocrit. Stroke 1988;19:1466-9.

2. Grolimund P, Seiler RW. Age dependence of the flow velocity in the basal arteries: A transcranial Doppler ultrasound study. Ultrasound Med Biol $1988 ; 14: 191-8$

3. Vriens EM, Kraaier V, Musbach M, Wieneke GH, Van Huffelen AC. Transcranial pulsed Doppler measurements of blood velocity in the middle cerebral artery: Reference values at rest and during hyperventilation in healthy volunteers in relation to age and sex. Ultrasound Med Biol 1989;15:1-8.

4. Macko RF, Ameriso SF, Akmal M, Paganini-Hill A, Mohler JG, Massry SG, et al. Arterial oxygen content and age are determinants of middle cerebral artery blood flow velocity. Stroke 1993;24:1025-8.

5. Brown MM, Wade JPH, Marshall J. Fundamental importance of arterial oxygen content in the determination of cerebral blood flow in man. Brain 
$1985 ; 108: 81-93$.

6. Doering T.J, Brix J, Schneider B, Rimpler M. Cerebral hemodynamies and cerebral metabolism during cold and warm stress. Am J Phys Med Rehab 1996; $75: 408-15$

7. Kuboyama T, Hori A, Sato T, Mikami T, Yamaki T, Ueda S. Changes in cerebral blood flow velocity in healthy young men during overnight sleep and while awake. Electroencephalography and Clin Neurophysiol 1997;102:125-31.

8. Klingelhofer J, Hajak G, Matzander G, Schulz-Varszegi M, Sander D, Ruther $\mathrm{E}$, et al. Dynamies of cerebral blood flow velocities during normal human sleep. Clin Neurol Neurosurg 1995;97:142-8.

9. Ameriso SF, Paganini-Hill A, Meiselman HJ, Fisher M. Correlates of middle cerebral artery blood velocity in the elderly. Stroke 1990;21:1579-83.

10. Arnolds BJ, von Reutern GM. Transcranial Doppler sonography. Examination technique and normal reference values. Ultrasound Med Biol 1986;12:115-23.

11. Tong DC, Albers GW. Normal values. In: Babikian VL, Wechsler LR, Toole JF, editors. Transcranial Doppler Ultrasonography. Butterworth Heinemann, $2^{\text {nd }}$ Ed. New York: Raven Press; 1999. p. 34.

12. Hudak ML, Koehler RC, Rosenberg AA, Traystman RJ, Jones MD. Effect of hematocrit on cerebral blood flow. Am J Physiol 1986;251:H63-70.

13. Massih J, Tang YL, Hudak ML, Koehler RC, Traystman RJ, Jones MD Jr. Effect of hematocrit on cerebral blood flow with induced polycythemia. J Appl Physiol 1987;62:1090-6.

14. Fiermonte G, Aloe SMA, Latagliata R, Petti MC, Giacomini P. Polycythaemia vera and cerebral blood flow: A preliminary study with transcranial Doppler. .J Int Med 1993;234:599-602.

15. Ameriso SF, Meiselman H, Saraj A, Fisher M. The effect of hemorheologic factors on middle cerebral artery blood flow velocity in young individuals. .J Neuroimaging 1992;2:16-8.

16. Sohn YH, Kim GW, Kim JS. Do hematocrit and serum fibrinogen influence transcranial Doppler measurements? J Korean Med Sci 1997;12:405-8.

17. Schuurman PR, Albrecht KW. Intraoperative changes of transcranial Doppler velocity: Relation to arterial oxygen content and whole-blood viscosity. Ultrasound Med Biol 1999;25:151-4.

Accepted on 01.12.2004

\section{Invited Comments}

In their study, Isikay et al have analyzed the influence of age and hematocrit on transcranial Doppler (TCD) parameters in two different clinical settings, i.e. acute ischemic stroke and non-vascular neurological disorders. Their chief conclusion is that changes in either age or hematocrit do not seem to modify blood flow velocities in patients with a recent ischemic stroke as much as they do in patients with other conditions. This may be of practical importance, since the assessment of cerebral hemodynamics in acute stroke is one of the main applications of TCD.

TCD is a non-invasive ultrasound technique that allows the measurement of blood flow velocities through the major basal intracranial arteries. During the last few years, it has turned out to be quite a useful tool for the evaluation of cerebral blood flow. In addition to its non-invasive character, TCD offers the advantages of being a rapid, reproducible, dynamic, and relatively inexpensive test. In patients with cerebral ischemia, TCD can detect intracranial arterial occlusions and stenoses, as well as the hemodynamic consequences of severe extracranial arterial lesions. TCD can also recognize microembolic signals coming from cardiac or vascular sources, while contrast-enhanced TCD can provide helpful information in right-to-left cardiac or extracardiac shunts. During the acute phase of cerebral ischemia, TCD monitoring can document spontaneous or tPA-induced arterial recanalization. Moreover, its efficacy as an auxiliary technique for the selection of patients for thrombolysis is currently being explored. Therefore TCD may assist the clinician in making the diagnosis, predicting the prognosis, and selecting the best treatment choice for patients with a recent ischemic stroke.

Despite its many advantages, TCD has several limitations.
Some of them are of technical nature, such as the lack of a suitable transtemporal window in a few patients, or the dependence of blood flow velocity measurements on the probeto-vessel insonation angle. Besides, a number of anatomic and physiologic factors are known to influence TCD parameters, such as the age and sex of the subject, the diameter of the vessels, some cardiovascular and gasometric variables, or the hematocrit value. Though the effects of these factors may at times be ignored, taking them into account can be crucial under certain circumstances. In accordance with previous reports, Isikay et al have found a relationship of TCD velocities with age and hematocrit: in general, the higher the age or the hematocrit, the lower the velocities recorded by TCD. However, the association of TCD velocities with both variables was more remarkable in the group of patients with non-vascular neurological disorders than in those patients suffering from acute ischemic stroke; in fact, the association between TCD velocities and hematocrit did not reach a significant level within the stroke group.

The results of this study are clinically relevant. If age and hematocrit do not have a great impact on TCD velocities in acute stroke, taking them under consideration may be unnecessary in this setting. Still, further research is needed to corroborate these findings in other patient series. It would also be interesting to elucidate the pathophysiological mechanisms that could potentially blunt the effects of age and hematocrit on blood flow velocities during acute cerebral ischemia.

María Luz Cuadrado

Department of Neurology, Rey Juan Carlos University, Madrid, Spain.E-mail: luz.cuadrado@urjc.es 